\title{
Research and Development of Nanopharmaceuticals in China
}

\author{
Changxiao Liu ${ }^{1}, 2^{*}$
}

\begin{abstract}
${ }^{1}$ Research Center of New Drug Evaluation, The State Key Laboratories of Pharmacodynamics and Pharmacokinetics, Tianjin Institute of Pharmaceutical Research, Tianjin 300193, China ${ }^{2}$ Research Center of Biological Evaluation of Nanopharmaceuticals, China National Academy of Nanotechnology and Engineering, Tianjin 300457, China
\end{abstract}

*Corresponding authors. Email: liuchangxiao@vip.163.com

\begin{abstract}
Nanopharmacoeuticals based on nanomaterials and nanotechnology are medicinal products for drug delivery, nano drugs and nano therapies, in vivo imaging, in vitro diagnostics, biomaterials, and active implants. Nanoscience and nanotechnology in China become ever more consequential in our lives; all members of the scientific community should better inform and educate the public about the great changes this new nano era is likely to bring. Here we review some main advances on the research and development of nanomaterials, nanotechnology and nanopharmaceuticals in China. For nanopharmaceuticals, we focus on the research and application of nanotechnology in anti-cancer drugs, and biological evaluation studies of nanomaterials.
\end{abstract}

Keywords: China; Nanomaterials; Nanotechnology; Nanopharmacoeuticals; Research

Citation: C. Liu, et al. Research and Development of Nanopharmaceuticals in China. Nano Biomed Eng. 2009, 1(1), 1-12. DOI: 10.5101/nbe.v1i1.p1-12

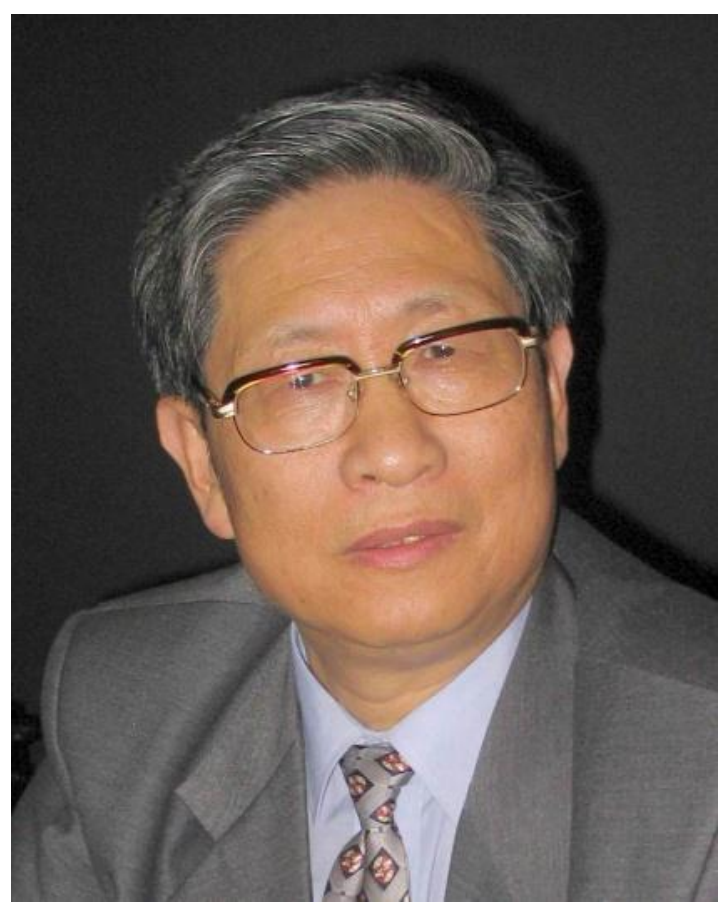

Professor Changxiao Liu is the Academician of Chinese Academy of Engineering, Doctorate advisor, research fellow. Liu was born in Yongxing County, Hunan Province in 1942, and graduated from Beijing Medical College (now Peking University). Liu is honorary president and director of Academic Committee of Tianjin Institute of Pharmaceutical Research, Director of Research Center for New Drug Evaluation and Director of National Key Laboratory of Pharmacokinetics and Pharmacodynamics at Tianjin Institute of Pharmaceutical Research, Director of Biomedical Evaluation of Nanomedicines, and National Institute of nanotechnology. Liu is Vice-Chairman of Chinese Pharmacological society, Chairman of Tianjin Pharmacological society, Chairman of Tianjin Pharmaceutical society. He is member of the Committee of Experts for four nanomedicine programs. In 1968, Liu set up the first pharmacokinetics laboratory in China. In 1980, he published the first book in China titled Drug Metabolism and Pharmacokinetics. In 1984, he published another new book, Introduction to Pharmacokinetics. Liu is the first among his Chinese peers to apply pharmacokinetics to the research of new drugs. Liu has been in charge $\mathrm{f} 28$ research projects for national key scientific and technological plan, new drug funds and pharmacokinetic study in key projects for National "863" and "973" Plans. Some projects have been listed as the national priority project for key scientific and technological plans, such as "study on the standardization for pharmacokinetics of prodrugs" and "study on the standardization for pharmacokinetics of bio-technical products". In 2003, Liu firstly propose metabonomics studies in China, and organized metabonomics studies of traditional Chinese medicines and bioinformation study. Two key projects on metabonomics have been finished in Tianjin and Zhejiang. Liu has been awarded numerous prizes in China, Thailand and 
Germany, such as National Science Conference Prize (1978), 30 terms of Scientific and Technical Achievement Prizes in China and local government (Tianjin, Beijing, Hunan and Jiangsu) and International Academic Prizes (1984-2002), including the first Redbud Prize for medical achievements (2000), Shimatzu Prize (1993), etc. Professor Liu was awarded advanced worker (1978, Hunan), the special government allowance by the State Council (1992), model worker (2000, Tianjin) and National model worker (2005) and Chinese Outstanding person (2000). He has published more than 280 papers and 16 books in Chinese and in English.

\section{Introduction}

Nanopharmacoeuticals based on nanoscience and nanotechnology are medicinal products for drug delivery, nanoscale drugs and therapies, in vivo imaging, in vitro diagnostics, biomaterials, and active implants. In the field of nanomedicine, these countries like USA, Japan, Canada, China, India, Korea, Russia, Singapore, and Switzerland firstly started the research on the drug delivery in early 1980-1990s by employing dendritic nano architecture for the controlled and targeted delivery of anticancer bioactivities. Many laboratories and institutions in these countries also initiated the research and development of nanopharmaceutical products since 1990s [1-15].

The science of nanopharmaceuticals exploits and builds upon novel research findings in the interdisciplinary area of nanotechnology, biology and medicine; it unifies the efforts of scientists, engineers, and physicians, with the aim of applying their latest research results to translational and clinical medicine by developing novel approaches and a better understanding of solutions to health-related issues, ultimately improving the quality of life. In medicine, improvements in targeted drug delivery, imaging, and therapy can lead to successful interventions to some diseases such as breast cancer, hepatitis, prostate cancer, etc.

However, nanopharmaceuticals, as currently pursued by the scientific community, use nanotechnology as an enabling technology to play an important part in the process of medical innovation. Tissue engineering and regenerative medicine are included as far as biomaterials are concerned. Nanopharmaceuticals will bring about enormous changes in medicine and treatment of patients. The promise and limitation of nanopharmaceutical tools, techniques, and materials in the context of unsolved medical problems will be explored. It will also address questions of sustainability, toxicity, ethics, societal and environmental impact. The debate will start with clinicians reporting the unsolved medical problems in the following fields: cardiology, oncology, neurology/neurosurgery, and dermatology, orphan diseases, orthopaedics and implants, haematology, inflammatory and infections diseases.

In recent years we have been watching the development of nanotechnology and nanopharma-ceuticals, and have paid special attention on its development of risk analysis, safety issues and biomedical evaluations [12-14]. This review will introduce some main information on China's recent research and development of nanopharmaceuticals based on nanoscience and nanotechnology.

\section{Nanoscience and nanotechnology in China}

Nanoscience and nanotechnology in China become ever more consequential in our lives. We, as the members of the scientific community, need to better inform and educate the public about the transformations this new nano era is likely to bring. Like many other countries, we expect that the development of nanoscience and nanotechnology will greatly affect many areas of scientific research and industrial development, and many aspects of our everyday life. Research, development and application of nanotechnology in China can be summed up in three characteristics: the first, China government's support for sustainable development; the second, significant academic achievements; the third, a clear consensus on sustainable development for nanoscience and nanotechnology [15].

\subsection{The government's support for sustainable de- velopment}

When the concept of nanoscience and nanotechnology was firstly introduced in the 1980s, it was received favorably in China. The initial interest was in part stimulated by the development of new tools and techniques, for example, scanning probe microscopes (SPM) which can be used for observing materials on the nanoscale. Soon after the concept began trickle through the scientific ranks, the Chinese Academy of Sciences, the National Natural Science Foundation Commission of China, and the State Science and Technology Commission (the Ministry of Science and Technology) began support nanoscience-related work and activities. China also has helped those who work in nanoscience and nanotechnology to develop their sense of being part of a new research and development community. For example, since 1990, dozens of international and domestic conferences in the field of nanoscience and nanotechnology have been held in China. These conferences addressed a wide range of topics in nanoscience and nanotechnology and attracted wide attention and public interests. In the 1990s, support for the development of nanoscience and nanotechnology increased substantially, largely by several major initiatives. For example, in 1990, State Science and Technology Commission of China launched the nearly decade-long "Climbing Up" project on nanomaterial science. In 1999, the Ministry of Science and Technology of China started a national basic research project ("973" Plan) entitled "Nanomaterial and Nanostructure", and has been providing budgets for basic research on nanomaterials such as nanotubes, quantum 
dots, nanosensor, etc. China National High Technology Plan ("863" Plan), which encompasses many categories of technology, has included a series of projects for nanomaterial applications. From 1990 to 2005 alone, over 1200 projects were implemented. In addition, during this period, the National Natural Science Foundation of China approved nearly 1000 small-scale projects associated with nanoscience and nanotechnology. With so much projects of the nano-related research and development going on in so many different places in China, in 2000, we set up the National Steering Committee for Nanoscience and Nanotechnology to oversee national policy and plans in these arenas. [13-15]

During the Ninth Five-year Plan period (19962000), the national "863" Plan supported by China government started the projects of improving nanobiotechnology. During the Tenth Five-ynaear period (2001-2005), national "863" and "973" Plans and the National Natural Science Foundation of China supported those researches of nanoscience, nanotechnology, and nanomedicines as priority subjects China spent 1500-2300 million RMB on nanotechnology research from 2001 to 2005 . It is estimated that about five percent of the budgets is spent on nanobiotechnology and nanomedicine related projects. During the Eleventh Five-year Plan period (2006-2010), the state is to increase markedly the support for nanoscience and nanotechnology research, China has spent 3000-4000 million RMB on nanotechnology research and development.

\subsection{Significant academic achievements}

Moving forward in nanoscience and nanotechnology requires a particularly wide spectrum of skills and knowledge. The demand for multidisciplinary research platforms with components assembled from academia and industry and that also have educational functions has become especially strong in recent years. According to incomplete statistics, more than 50 universities, 50 institutes and over 300 industry enterprises in China have engaged in nanoscience and nanotechnology research and development, involving in more than 3000 researchers across China. In order to move forward and become more competitive in nanoscience and nanotechnology, China needs to continue to expand its nowlimited research infrastructure. In some areas such as nanoscale devices with novel electronic and optoelectronic features, the efforts to consolidate resources to tackle key technological issues are under way. Efforts have also been made to pursue industrial-scale production of nanomaterials, such as carbon nanotubes, polymeric nanocomposites, and nanoparticle materials, with the intention of opening up opportunities for new businesses to sprout and grow. The nanoscience and nanotechnology community in China has made remarkable advances across the research and development spectrum, from fundamental scientific research to research- es associated with the potential societal applications of new nanotechnologies. China still has a long way to go to improve the overall competitiveness of its nanoscience and nanotechnology enterprise [12-15].

The scientific output of Chinese scientists in the field of nano research is becoming ever more and more significant. According to the Scientific Citation Index, the Chinese Academy of Sciences ranked fourth in the world in total number of citations among those institutions and universities that published more than 100 nanotechnology papers from 1992 to 2002. Another recent analysis of nanoscience productivity around the world ranked China at the top for the first 8 months of 2004. This should not be the reason for Chinese research community to be over-optimistic, however. The volume of published papers is only one of indicators of the value of research. Another available indicator is the impact factor, or the number of citations per paper. From 2001 to 2003, the number of citations per nanotechnology paper published by scientists in the United States, Germany, Japan, and China was about 6.56, 4.54, 3.7, and 2.28, respectively [15].

Since 2006, the total number of Chinese basic research papers on nanoscience and nanotechnology and total number of citations have put the second position in the world; tightly follow after the United States. According to incomplete statistical data from 2004-2008, Chinese scholars also published a large number of nano-research papers (more than 1000) in Chinese academic journals.

Nanobiotechnology in Hong Kong, an interdisciplinary science, combines physical laws, chemical procedures and biological principles together at the nanoscale. The recent establishment of the nanobiotechnology group in the University of Hong Kong has been looked as the first step to speed up the research in this field. The group has identified eight topics in which the University has approved research strengths, and will be seeking to develop these topics to internationallycompetitive levels. Four teams have been looking into DNA electronics, microfluidic devices for disease monitoring, the use of nano-metals/metal compounds for clinical applications, and the use of bio-polymeric materials for drug delivery. The fifth team is to develop techniques to exploit magnetic nanoparticles by using them as contrast agents in magnetic resonance imaging; and using lanthanide oxide nanoparticles as probes to detect bio-macromolecules. The sixth team is developing nanoindentation technologies for measuring the microstructures of biological tissues. The seventh team is investigating cellular and subcellular mechanics. Finally, the eighth team is exploiting recent advances in electrospinning technology to developing nano- and micro-fibers for tissue engineering applications. With the fast advances of inter-department and international collaboration, we hope to promote and expand nanomedicine in our community [3]. 
2.3 Sustainable development in nanoscience and nanotechnology

Facing the arduous difficulties in nanoscience and nanotechnology research, and the risks of nanomedicine/nanopharmaceutical industry, I suggest that we should pay attention on the four-oriented development in the area, according to China's national conditions. The first is the practice research-oriented, combining basic research with application. The second is to set up different professional disciplines for the research bases and to strengthen the efficacy, safety, and the industrialization, and feasibility study of nanopharmaceuticals in order to ensure sustainable development. The third is to focus on solving the challenging problem of the difficult implementation, and breakthroughs in nanoscience and nanotechnology. The fourth is to investigate the complexity in research and development of the new technologies required to support with long-term development, and to know the risks for technological transformation into industrialization [13].

\section{Advances on research and devel- oppment}

\subsection{Considerations for applications of nanopharma- ceuticals}

According to the data associated with the application research of nanotechnology in medicines, the most active area in medical research mainly focused on anticancer drugs, cardiovascular drugs and nervous system drugs.

Anti-cancer drugs: With regard to cancer diagnostics and treatment, nanotechnology-based in vivo diagnostic approaches and nanotechnology-based drug delivery systems (NDDS) are under development. For example, nanotechnology-based cancer diagnostics are developed with the aim of monitoring the therapeutic effects of drugs. Such a therapy-specific monitoring is expected to prove the efficacy of a drug within days compared to weeks or months with currently available diagnostic methods. For cancer therapies, some effective treatment drugs are found. The important research direction in nanomedicine is the development of NDDS for anticancer drugs that result in drug accumulation at the tumour site and in that way reduce side effects. This is due to the fact that medicine nanotechnology aims to improve and optimize material properties for their interaction with cells and tissues, to allow passive tumor targeting, crossing the blood-brain barrier, or to improve the bioavailability.

NDDS are a sub-class of advanced drug delivery systems that consists of drug carriers. Examples for NDDS are liposomes, nanosuspensions, polymeric nanoparticles, dendrimers, fullerenes, carbon nanotubes, and inorganic nanoparticles. According to experts, the benefit of NDDS for the patient will be less side-effects and improved efficacy of drugs, and the possibility to treat diseases and disease stages that currently cannot be treated with conventional drugs. The early development stage of NDDS based on nanotechnology is to increase the solubility or to concentrate drugs in the diseased tissues by physical effects. Up to date, the products will try to tailor pharmacokinetic properties of drugs and therewith also enhance their efficacy. Further, the whole research field of NDDS is still in its infancy compared with small molecule drugs or even biologics to increase the efficacy of drugs.

Cardiovascular drugs: Cardiovascular diseases are the leading cause of death in the world with $30 \%$ of the projected death ratio. Due to the high number of patients with cardiovascular diseases, even small cost effects on an individual case basis will result in large cost impacts, if the whole affected population is taken into consideration. Some nanotechnology-based diagnostic tests for stroke patients may offer a potential for cost reductions. Effective prevention strategies for these high risk patients could result in reduced costs for intensive care and rehabilitation for stroke patients. The contrast agent is based on nanoscale perfluorocarbon emulsion particles and is currently in a preclinical development stage.

Nervous system drugs: In the diagnostic group of nervous system diseases, NDDS are in the course of development, and are able to transport drugs across the blood-brain barrier.

\subsection{Achievements in research of nanopharmaceuti- cals in China}

Nanotechnologies, especially nanomedicine including nanopharmaceuticals, are advancing significantly day by day. Nanoparticles are being already used in many products (mainly in cosmetics), but other spheres such as pharmaceutics and general medicine are slowly applying nanotechnology standards. The key issues of druggablity of nanopharmaceuticals are drug targeting and safety. The drug delivery system is to make the life-saving drug available in that part of the body where it is required the most. However, most of the time, these systems fail to work efficiently because the particles of the drug are too large for the cells to absorb, or they are insoluble or they have the potential to cause tissue damage. Due to their exceedingly small size, nanoparticles are easily taken up by the cell. Moreover, they are completely soluble and they do not also damage the tissues. Nanoscience and nanotechnology and their applications in China have developed rapidly. From basic to application, Chinese researchers have published a lot of papers $[11,16-24,36-43,45-50,52]$. However, the number of nanopharmaceutical research papers are still not too many, limited progress have made in the new promising applications of nanotechnology research and development. From the limited literature information, the advances of nanotechnology research and applica- 
tion in anti-cancer drugs and biological evaluation studies are also reviewed herein.

\subsubsection{Biopharmaceutics and therapeutic potential of engineered nanomaterials}

Engineered nanomaterials are at the leading edge of the rapidly developing nanosciences, and are an important class of new materials with specific physicochemical properties different from bulk materials with the same compositions. The potential of nanomaterials is rapidly expanding with novel applications constantly being explored in different areas. The unique sizedependent properties of nanomaterials make them very attractive for pharmaceutical applications. Investigations of physical, chemical and biological properties of engineered nanomaterials have yielded very valuable information. Cytotoxic effects of certain engineered nanomaterials towards malignant cells have become the basis for one aspect of nanomedicine. It is inferred that size, three dimensional shape, hydrophobicity and electronic configurations make them appealing subjects in medicinal chemistry. Their unique structures coupled with immense number of derivatizations provide a solid basis for exciting developments in therapeutics.

It has made progress to address the fate of absorption, distribution, metabolism and excretion (ADME) of engineered nanoparticles in vitro and in vivo. It updates the distinctive methodology used for studying the biopharmaceutics of nanoparticles. Liang et al's review addressed the future potential and safety concerns and genotoxicity of nanoparticle formulations in general. It particularly emphasizes the effects of nanoparticles on metabolic enzymes as well as the parenteral or inhalation administration routes. Herein I focus on the potential of nanomedicine by discussing biopharmaceutics of fullerene derivatives and their suitability for diagnostic and therapeutic purposes. Future direction is discussed as well [11].

\subsubsection{Bio-distribution and biodegradation}

Drug targeting distribution of nanopharmaceuticals is an impart ant for selecting pharmacodynamic and pharmacokinetic charasitictcs. Cytostatics are bonded to the iron oxides. This form of administration, also known as sluicing, is particularly suitable for cytostatics, since the intention is to achieve a high concentration of the cytostatic at the target site (site of the tumor), but to minimize the harmful effect in the rest of the tissue [35] . The toxicity of currently available anticancer drugs and the inefficiency of chemotherapeutic treatments, especially for advanced stages of the disease, have limited the optimization of clinical drug combinations and effective chemotherapeutic protocols. Nanomedicine allows the release of drugs by biodegradation and self-regulation of nanomaterials in vitro and in vivo. Nanotechnologies are characterized by effective drug encapsulation, controllable self-assembly, specificity and biocompatibility as a result of their own material properties. Nanotechnology has the potential to overcome current chemotherapeutic barriers in cancer treatment, because of the unique nanoscale size and distinctive bioeffects of nanomaterials. Nanotechnology may help to solve the problems associated with traditional chemotherapy and multidrug resistance [16].

Successful development of safe and effective nanoprobes for tumor targeting and selective therapy is challenging task. In recent years, gold nanorods (GNRs) have attracted considerable attention for their unique properties in photothermal therapy [36] Cui et al's study showed highly selective targeting and destructive effects on the cancer cells and solid tumors under nearinfrared laser irradiation. Mouse models loaded with melanoma A375 cells were established, and the biodistribution of RGD-dGNRs was showed in this model (Figure 1). They successfully observed the disappearance of tumors implanted in four sample mice from test group of ten. High-performance RGD-conjugated dendrimer-modified GNR nanoprobes exhibit great potential in applications such as tumor targeting, imaging, and selective photothermal therapy [37].

With the rapid development of quantum dot (QD) technology, water-soluble QDs have the prospect of being used as a biological probe for specific diagnoses, but their biological behaviors in vivo are little known. Our recent in vivo studies concentrated on the biokinetics of QDs coated by hydroxyl group modified silica networks (the QDs are 21.3 $\pm 2.0 \mathrm{~nm}$ in diameter and have maximal emission at $570 \mathrm{~nm}$ ). Male ICR mice were intravenously given the water-soluble QDs with a single dose of $5 \mathrm{nmol} / \mathrm{mouse}$. Inductively coupled plasma-mass spectrometry was used to measure the (111) Cd content to indicate the concentration of QDs in plasma, organs, and excretion samples collected at predetermined time intervals. Meanwhile, the distribution and aggregation state of QDs in tissues were also investigated by pathological examination and differential centrifugation. The plasma half-life and clearance of QDs were 19.8 $\pm 3.2 \mathrm{~h}$ and $57.3 \pm 9.2 \mathrm{~mL} \cdot \mathrm{h}^{-1} \cdot \mathrm{kg}^{-1}$, respectively. The liver and kidney were the main target organs for QDs. The QDs metabolized in three paths depending on their distinct aggregated states in vivo. A fraction of free QDs, maintaining their original form, could be filtered by glomerular capillaries and excreted via urine as small molecules within five days. Most QDs bound to protein and aggregated into larger particles that were metabolized in the liver and excreted via feces in vivo. After five days, $8.6 \%$ of the injected dose of aggregated QDs still remained in hepatic tissues and it was difficult to clear this fraction of QDs [42].

Liang et al presented that the future potential and safety concerns and genotoxicity of nanoparticle formulations in general. It particularly emphasizes the effects of nanoparticles on metabolic enzymes as well 
A

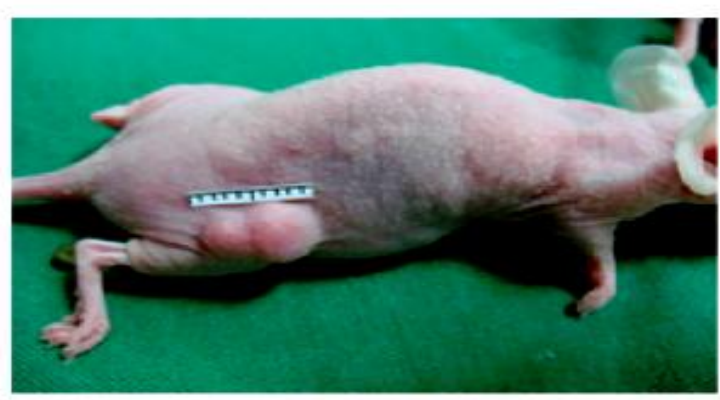

C

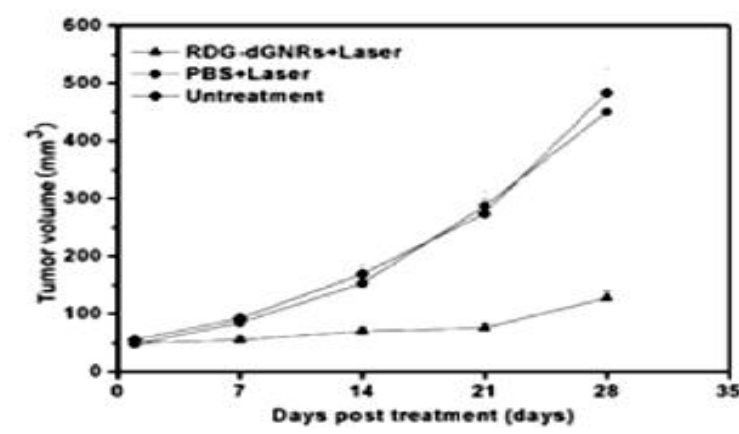

B

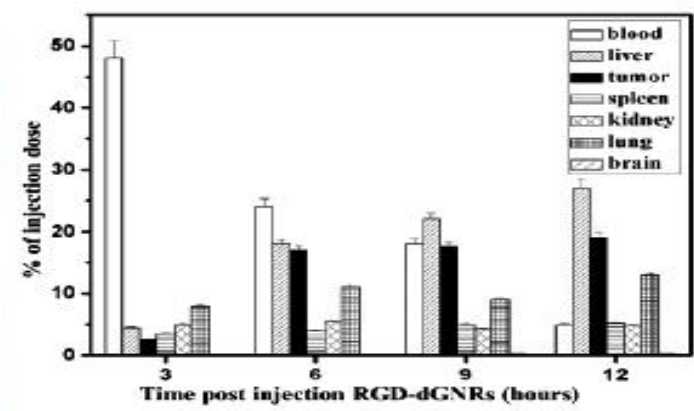

D

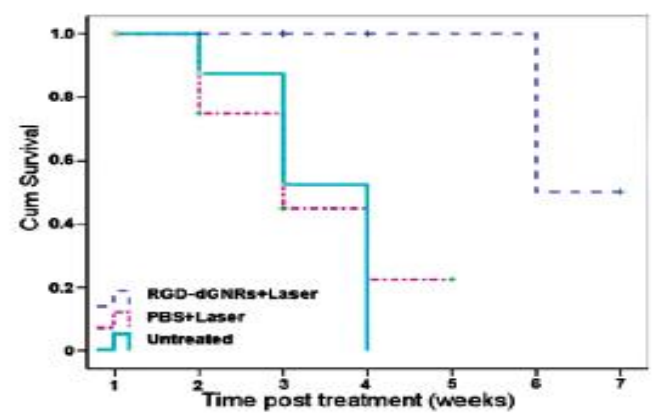

Figure 1. Melanoma animal models, biodistribution of RGD-dGNRs, and survival data analysis of control and test group [37] (A) A375 melanoma mouse models. The tumor size can be calculated as ab2/2 (a represents the longer dimension and $\mathrm{b}$ represents the shorter dimension of the tumor). (B) Biodistribution of RGD-dGNRs in mice after intravenous injection. Several time points after injection, gold amounts in tissue samples were evaluated by ICP mass spectrometry. (C) Tumor size at different time points postirradiation of mice treated with RGD-dGNRs plus NIR laser (group 1); PBS plus NIR laser (group 2) or untreated control (group 3), P < 0.05 for group 2 or group 3 versus group 1. (D) Kaplan-Meier curve of the test group and control group

as the parenteral or inhalation administration routes of nanoparticle formulations [30].

\subsubsection{Doxorubicin PEG-PE-nanoparticles}

Solid tumors account for more than $85 \%$ of cancer mortality. To obtain nutrients for growth and metastasis, cancer cells must grow around existing vessels or stimulate formation of new blood vessels. These new vessels are abnormal in structure and characterized by leakage, tortuousness, dilation, and a haphazard pattern of interconnection. Tumor structure and blood flow hinder the treatment of solid tumors. To reach cancer cells in optimal quantity, a therapeutic agent must pass through an imperfect blood vasculature to the tumor, cross vessel walls into the interstitium and penetrate multiple layers of solid tumor cells. Recent studies have demonstrated that poor penetration and limited distribution of doxorubicin in solid tumors are the main causes of its inadequacy as a chemotherapeutic agent.

Liang $\mathrm{W}$ et al research results on doxorubicincontaining PEG-PE micelles are an important contribution to nanomedicine development (which is called "nanoparticles carry chemotherapy drug deeper into solid tumors"). Encapsulation of doxorubicin into PEG-PE micelles increased its accumulation and penetration in tumors in terms of both the percentage of cells that were reached by the drug and the intracellular levels that were attained. This increased accumulation and penetration can be attributed to the efficient internalization of the drug-containing micelles by the endocytotic cell uptake mechanism and enhanced permeability and retention of tumors with leaky vasculature. High intracellular retention is especially important because doxorubicin must be internalized into tumor cells to realize the effective therapy for tumors. The doxorubicincontaining PEG-PE micelles had greatly increased antitumor activity in both subcutaneous and lung metastatic LLC tumor models compared with free doxorubicin. However, mice treated micelle- encapsulated doxorubicin showed fewer signs of toxicity than those treated with free doxorubicin. This drug packaging technology may provide a new strategy for design of cancer therapies [43]. J Natl Cancer Inst gave a high evaluation for their research [44].

Wei et al carried out study on comparative pharmacokinetics of nanoparticle mic3lle-encapsulated doxorubicin (M-Dox), liposome encapsulated doxorubicin (L-Dox0 with general doxorubicin (G-Dox). Their results indicated that M-Dox had similar pharmacokinetic characteristics to G-Dox, but L-Dox was significant 
different from G-Dox, which had smaller Vd and CL, and higher AUC based on plasma level than those for G-Dox [45]. Our laboratory studied the accumulation of doxorubicin nanoparticles in tissues of tumor-bearing mice. Compared with general doxorubicin preparation, which is a marketed product, nanoparticle micelle of doxorubicin has the similar pharmacokinetics in the tissue, and the similar concentrations in the tumor tissue. However, the accumulation of doxorubicin in the heart, spleen, kidney, lung, tumor, muscle and skin decreased significantly after three intravenous injections, showing that the nanomicelle can improve the elimination of doxorubicin in most tissues. It is deduced that the sideeffects of doxorubicin after clinical use may be reduced significantly [46].

\subsubsection{Vinorelbine nanomicelle}

Vinorelbine is a vinca-alkaloid obtained by chemical semi synthesis. Vinorelbine inhibits microtubule assembly, and thus its activity is cell cycle specific. Clinically, vinorelbine is a potent anti-cancer drug widely prescribed for advanced non-small-cell lung cancer and advanced breast cancer. Like most of conventional chemotherapeutic agents, vinorelbine is distributed nonspecifically in the body where it affect both cancerous and normal cells, thereby resulting in excessive toxicities and limiting the dose achievable within the tumor. The novel LC-ESI/MS and LC-ESI/MS/MS methods for the quantification of vinorelbine in biosamples have been developed and fully validated. The thesis aimed to study the preclinical pharmacokinetics of vinorelbine nanomicelle (M-VRL) and free vinorelbine (F-VRL) in rats and mices by LC-ESI/MS and LC-ESI/MS/MS, to exactly predict the transformation of vinorelbine in human, to rationally evaluate the efficiency and security of vinorelbine, and to offer some references and inspirations for the clinical studies [47]. Nanomicelle, by using both passive and active targeting strategies, can enhance the efficacy and reduce the toxicity of antitumor agents.

\subsubsection{Paclitaxel magnetoliposomes}

Paclitaxel, belonging to the taxane class of anticancer agents, perhaps is the most important chemotherapeutic agent against cancer over several decades. In clinical trials, paclitaxel has been successfully used for the treatment of ovarian, breast, lung, and head/neck cancers and AID Srelated Kaposi sarcoma. To improve the water solubility of paclitaxel and explore the best type of paclitaxel liposomes, lyophilized paclitaxelloaded negatively charged and magnetic liposomes were evaluated for their preferential presentation to the tumor under neoplastic condition, and enhanced drug level in tumor and plasma, as well as decrease of drug uptake in heart, liver, and spleen.

Zhang et al reported the results on in vitro and biological evaluation of lyophilized negatively charged paclitaxel magnetic liposomes as a potential carrier for breast carcinoma via parenteral administration [48]. Pharmacokinetic studies showed that encapsulation of paclitaxel in magnetoliposomes produced marked difference over the drug in Cremophor EL/ethanol pharmacokinetics, with an increased $\mathrm{t}_{1 / 2} 19.37 \mathrm{~h}$ against 4.11 h. For in vivo distribution, paclitaxel concentration of lyophilized magnetoliposomes in the tumor was much higher than that of lyophilized conventional liposomes or Cremophor EL/ethanol, whereas in heart it was much lower than the latter two formulations via s.c. and i.v. administration. Lyophilized paclitaxel magnetic liposomes showed more potency on the therapy of breast cancer than other formulations via s.c. and i.p. administration. This study demonstrates that paclitaxel magnetoliposomes can effectively be delivered to tumor and exert a significant anticancer activity with fewer side effects in the xenograft model.

Cui et al successfully prepared anti-HIF-1a antibody-conjugated NMs filled with paclitaxel (PTX), and confirmed the prepared nano-drugs can target specifically and selectively kill MGC-803 cancer cells, markedly decrease the toxic side-effects of PTX. Because of intensive expression of HIF-1a in almost all cancer cells, and Pluronic P123 polymer being capable of inhibiting MDR, this unique nanopharmaceuticals, anti-HIF-1a antibody-conjugated NMs filled with PTX, exhibit attracting technological prospect, and have great potential in tumor molecular imaging, and targeting therapy in near future [41].

\subsubsection{Micelle-Encapsulated Alprostadil}

Alprostadil (APL) is endogenous substance that is able to dilate vessels, to inhibit platelet aggregation, therefore, potentially be active for the treatment of chronic arterial obstruction and microcirculation disturbance. However, because of its weak stability in biological matrixes, new delivery systems such as nanoparticle micelle needed to be designed. Li et al studied the pharmacokinetics and tissue distribution of micelle encapsulated alprostadil (M-Alp) and free alprostadil (FAlp) by intravenous injection of $200 \mu \mathrm{g} / \mathrm{kg}$ in rats. The pharmacokinetic results showed that the Apl was eliminated quickly with half-life time of 4.39 min and 4.76 min, for M-Apl and F-Apl, respectively. The tissue distribution results indicated that the novel M-Apl tended to accumulate into tissues such as heart, liver etc. This nanocarrier assembling technology may provide a new strategy for delivery of Apl for the treatment of chronic arterial obstruction and microcirculation disturbance [49].

\section{Challenges of research and develop- ment of nanopharmaceuticals}

\subsection{Risk issues}

The introduction of nanomedicine including nanopharmaceuticals into clinical practice has already produced 


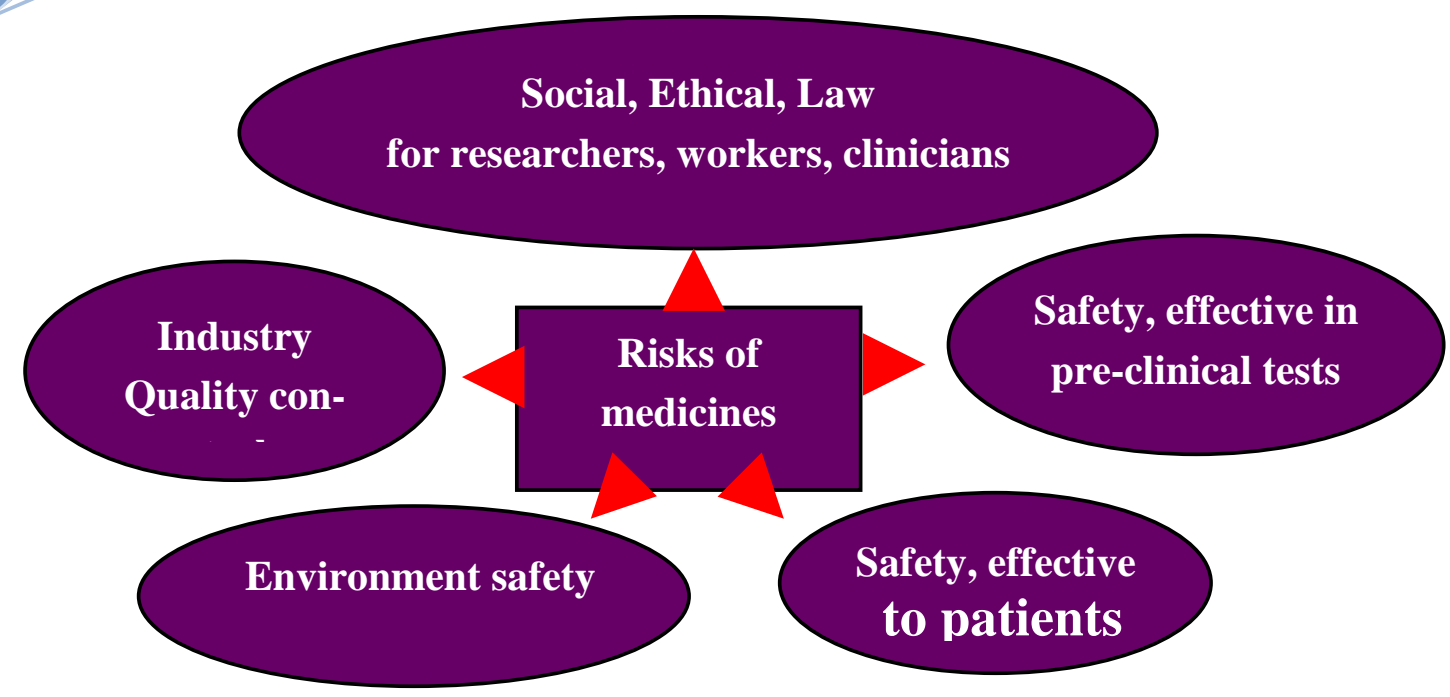

Figure 2. The risks of nanomedicine development

its first successes. Beginning with the basics, the subject is developed to potential clinical applications, many of which are still at an experimental stage. However, its use in humans is only possible in a few areas today, and numerous hurdles still have to be overcome through the research and development of outstanding solutions. Important components of nanomedicine such as nanodiagnostics and nanopharmaceuticals are covered extensively.

Many nonomedical scientists, pharmaceutical personnel, as well as physicians, hope that the descriptions of the technology involved and other medical terminology are kept as clear and simple as possible. Due to the early stage development of nanotechnology, these are mainly deliberations about possible social and ethical issues for nanotechnology in general. The majority of these analyses relate to nanotechnology in their entirety, the different analysis of certain application areas or subfields of nanotechnology or of specific product lines has just started recently. Generally two forms of application of nanoparticles need to be distinguished with respect to exposure and possible health risks: (1) Nanoparticles bound into a chemical matrix, such as a polymer or a metal. Unless nanoparticles are released due to chemical processes, these materials are generally considered as safe; and (2) Free nanoparticles in the air or in fluids that can be taken up by the body via the lung, skin or the intestinal tract. To obtain market approval, these regulations require a risk assessment and management, whereas the regulatory requirements must be sufficiently rigid to ensure safety and quality of the medicinal products.

As like Figure 2, the risks of development assessment of nanomedicine mainly come from the following aspects: (1) social, ethical and law issues, (2) environment issues, (3) industry issues, (4) experiment in safety and effective to animals and (5) human health in medical use.

\subsection{The research areas of pharmaceutical sciences based on nanotechnology}

Nanomedicines represent an emerging field where the nanoscale element may refer to either the size of the drug particle or to a therapeutic delivery system. These therapeutic systems may be defined as a complex system consisting of at least two components, one of which is the active ingredient. In this field the concept of nanoscale is the range from 1 to $1,000 \mathrm{~nm}$. The definition includes polymer therapeutics, which share many characteristics with macromolecular prodrugs such as antibody-conjugates drugs. Nanomedicines can be developed either as drug delivery systems or biologically active drug products. The areas in pharmaceutical sciences that use nanotechnology includes the following aspects: drug discovery, including combinatorial chemistry and synthesis on the molecular and macromolecular scale; nanoanalysis, including bioanalysis using miniaturized probes, microarrays, and lab-on-a-chip approaches; body fluid approaches; drug delivery systems, such as liposomes, nanoparticles, microemulsions, dendrimers, having sizes in the nanometer range; implantable devices that can sense blood levels and automatically administer drugs; nanoscale biomaterials including biomimetic materials; biological macromolecules, such as proteins, enzymes, DNA-and RNA-based nanostructures, molecular assemblies, biomolecules, cells, biochips; molecular sensors and biosensors, clinical diagnostic techniques; and gene delivery and expression.

\subsection{The research areas in biomedical evaluation of nanopharmaceuticals}


Nanotechnology manifests that itself can be used for medical application in a wide range of materials. The unprecedented freedom to design and modify nanomaterials to target cells, chaperone drugs, imaging biomolecular processes, sense and signal molecular responses to therapeutic agents, and guide surgical procedures is the fundamental capability offered by nanotechnology, which promises to impact drug development, medical diagnostics, and clinical applications profoundly [11-13].

Engineered nanomaterials are at the leading edge of the rapidly developing nanosciences and are founding an important class of new materials with specific physicochemical properties different from bulk materials with the same compositions. The potential for nanomaterials is rapidly expanding with novel applications constantly being explored in different areas. The unique size-dependent properties of nanomaterials make them very attractive for pharmaceutical applications. Investigations of physical, chemical and biological properties of engineered nanomaterials have yielded valuable information. Cytotoxic effects of certain engineered nanomaterials towards malignant cells form the basis for one aspect of nanomedicine. It is inferred that size, three dimensional shape, hydrophobicity and electronic configurations make them an appealing subject in medicinal chemistry. Their unique structure coupled with immense scope for derivation forms a base for exciting developments in therapeutics. This review article addresses the fate of absorption, distribution, metabolism and excretion of engineered nanoparticles in vitro and in vivo. It updates the distinctive methodology used for studying the biopharmaceutics of nanoparticles.

Up to date, scientists must accept this view that it is still very early in the toxicological evaluation for nanomaterials, nanomedicine and nanopharmaceuticals. The basic tenet of study designed to develop a study system of toxic effects of nanomaterials, nanomedicines/nanopharmaceuticals on biological systems is to understand the physico-chemical characteristics of nanomaterials, and nanopharmaceuticals. Therefore, the approach to address the safety and toxicity of these products will best be conducted via multidisciplinary terms. Many traditional methods and approaches will likely be applicable to study of nanopharmaceuticals.

Nanotechnology research and development is directed toward understanding and creating improved materials, devices, and systems that exploit these properties. In a review, Thomas et al reviewed that a limited subset of products that contain nanoscale materials, assess the available data for evaluating the consume exposures and potential hazard associated with these products, and discuss the capacity of US regulatory agencies to address the potential risks associated with these products [11]. Some of the potential impacts of dermal exposure to nanoscale materials include the following:
(1) enhanced amount and depth of penetration of active ingredients in cosmetic into the skin resulting in increased activity, (2) ingredients that are chemically unstable in air and light (as retinal and vitamin E) may be more readily used in topical products following encapsulation in nanoparticles, and (3) and timed release of ingredients may become more feasible in topical products and could allow for improved effectiveness equivalent to current controlled release orally administered drugs.

Due to the nanotechnology combines with biotechnology, a newly emerging cross-disciplinary field nanobiotechnology becomes the new developing area. As the research and application of nanotechnology, studying and understanding the complex relationship between nanopharmaceuticals and biological system will show special important to environmental, human health and safety. Criticism of the use of laboratory animals for the safety testing of chemicals is increasing, in society as a whole and also in the scientific world. This criticism is not only limited to ethical concerns, but scientific considerations also play a significant role. It should be realized that the animal bioassays presently used in toxicity testing are model systems for the prediction of toxicity in humans or the environment. In the last few decades new technologies and new knowledge have become available. This development is the result of toxicological research and the implementation of new methods and technologies [12-14, 50-51].

In nanotechnology research and development, science and bio-medical evaluation regardless of the environmental health of pre-clinical efficacy and safety, or on the efficacy and safety of patients are very necessary. According to peculiarities of nanopharmaceutical knowledge and our laboratory conditions, As Table1, we propose the following evaluation of research projects and contents include biodistribution, metabolic fate, persistance of non-degradable systems, specific therapeutic issues and immunogenicity.

A dedicated nanotoxicological risk assessment might be necesssary for novel nanopharmaceutical products which should take into account: (1) The biological fate of nanoparticles inlcuding distribution, accumulation and metabolism, (2) Medication-specific uptake routes related to the different routes of administration and the types of nanomaterials used, and (3) Possible side effects, caused by the interaction of nanoparticles with living matter or their transport across biological barriers. Toxicologists warn that health and environmental effects of nanoparticles and exposure of workers need to be investigated. There is consensus among stakeholders that the present level of knowledge is insufficient for risk assessments. The need to (1) Develop harmonised nomenclature and criteria for nanoparticle characterisation, (2) Develop methodologies for routine measurements, (3) Develop equipment and 
Table 1. The biomedical evaluation of nanopharmaceuticals

Evaluation terms Evaluation contents

Biodistribution

Metabolism and pharmacokinetics

Immunogenicity, immunopharmacology

Persistance of non-degradable systems

Biocompatibility

Specific therapeutic and toxicological issues
Whole organism, cellular level

Absorption, distribution, metabolism and excretion

$\mathrm{IgG} / \mathrm{IgM}$ production, cytokine induction

Possibility of lysosomal storage disease

Biological environment and toxicology and adverse effect to patients

Therapeutic index of nanomedicines and its delivery systems in drug delivery relates to toxicity pf drug payload methods for the determination of the environmental fate of nanoparticles and for the detection in the environment, and for exposure assessment to nanoparticles, (4) Understand toxicity of nanoparticles, and (5) Conduct epidemiological studies is broadly recognized.

\section{Concluding Remark}

The nascent field of nanopharmaceuticals science presents an exciting vision of the future. The ultimate goal of this field is to develop novel medical products based on nanomaterials and nanotechnology. Exploring nanopharmaceuticals may lead to understanding of fundamental laws and principles in medical chemistry, biodistribution, metabolism, toxicology, and interaction between nanomaterials-based nanodrugs and cells or tissues, organs. This new knowledge will enable the design and fabrication of a vast variety of nanodrugs and nano delivery systems for early disease diagnosis and therapy [52].

Nanomaterials and nanotechnology have exhibited great markets prospects for commercialization. For example, global market for drug delivery products amounts to U.S. dollar 33 billion per year, with global market for implants up to U. S. dollar 2 billion per year. It is estimated that products with nanoscale features contribute to around $1 \%$ of this total at present, and with the rapid dev elopement and potential benefits of nanobiotechnology, this percentage will grow exponentially in the near future. With increasing demand for better health treatments to improve the lifestyle and counter degenerative diseases of the industrialized world, the driving forces for research and development leading to new and better products are obvious. nanopharmaceuticals science based on nanomaterials and nanotechnology will grow quickly, nanoscale medical products will likely improve our healthcare soon.

\section{References}

1. Foldvari M. Nanomedicine research in Canada. Nanomedicine 2006; 2(4): 296

2. Zhao XJ. Nanomedicine Research in China. Nanomedicine 2006; 2(4): 297

3. Wong K. Development of nanomedicine in Hong Kong. Nanomedicine 2006; 2(4): 297

4. Jain NK. Status of nanomedicine research in India. Nanomedicine 2006; 2(4): 297

5. Urisu T, Wei CM. America-Japan Nanomedicine Society (AJNS). Nanomedicine 2006; 2(4): 297-298

6. Lee YS. Current research status of biomedical micro and nano technologies in Korea. Nanomedicine 2006; 2(4): 298

7. Yaminsky I. Outlook into the nanomedicine research in Russia. Nanomedicine 2006; 2(4): 298

8. Yun J. Singapore - an ideal R\&D hub for bionanotechnology. Nanomedicine 2006; 2(4): 299

9. Hunziker P. Nanomedicine Research in Switzerland. Nanomedicine 2006; 2(4): 299

10. Donald A. Tomalia. International report on nanomedicine in the U.S.A. Nanomedicine 2006; 2(4): 299

11. Liang XJ, Chen C, Zhao Y, Jia L, Wang PC. Biopharmaceutics and therapeutic potential of engineered nanomaterials. Curr. Drug Meta. 2008; 9(8):697-709. doi:10.2174/138 920008786049230

12. Si DY, Sun YD, Cheng TF, Liu CX. Biomedical evaluation of nanomedicines, Asian J. Pharmacodyn Pharmacokinet, 2007, 7(2):83-97

13. Sun YD, Chen ZM, Wei H, Liu CX. Nanotechnology challenge: safety of nanomaterials and nanomedicines. Asian $J$. Pharmacodyn Pharmacokinet 2007; 7(1): 17-31.

14. Cheng TF, Sun YD, Si DY, Liu CX. Attention on research of pharmacology and toxicology of nanomedicines. Asian $J$. Pharmacodyn Pharmacokinet 2009; 9(1):25-27.

15. Bai CL. Ascent of Nanoscience in China. Science 2005; 309: 61-63. doi:10.1126/science. 1115172

16. Liang XJ, Chen C, Zhao Y, Wang PC. Circumventing tumor resistance to chemotherapy by nanotechnology. Methods Mol. Biol. 2010; 596:467-88. doi:10.1007/978-160761-416-6_21

17. Liang F, Chen B.A Review on Biomedical Applications of Single-Walled Carbon Nanotubes. Curr. Med. Chem. 2009 Nov 24. [Epub ahead of print] 
18. Jia X, Fan H, Zhang F, Qin L. Using sonochemistry for the fabrication of hollow $\mathrm{ZnO}$ microspheres. Ultrason Sonochem 2010; 17(2): 284-7. doi:10.1016/j.ultsonch.2 009.09 .010

19. Ting G, Chang CH, Wang HE. Cancer nanotargeted radiopharmaceuticals for tumor imaging and therapy.Anticancer Res. 2009; 29(10):4107-18.

20. Li L, Liu J, Diao Z, Shu D, Guo P, Shen G. Evaluation of specific delivery of chimeric phi29 pRNA/siRNA nanoparticles to multiple tumor cells. Mo.l Biosyst. 2009; 5(11):1361-8. doi:10.1039/b903428e

21. Liu L, Zhang J, Su X, Mason RP. In vitro and In vivo Assessment of CdTe and CdHgTe Toxicity and Clearance. $J$. Biomed. Nanotechnol. 2008; 4(4): 524-528. doi:10.11 66/jbn.2008.018

22. Wang Y, Tu SL, Li RS, Yang XY, Liu LR, Zhang QQ. Cholesterol succinyl chitosan anchored liposomes: preparation, characterization, physical stability, and drug release behavior. Nanomedicine 2009 Oct 2.

23. Gao J. MRI-Visible Micellar Nanomedicine for Targeted Drug Delivery to Lung Cancer Cells. Mol. Pharm. 2009 Aug 26.

24. Huang H, Chen M, Bruno P, Lam R, Robinson E, Gruen D, Ho D. Ultrananocrystalline diamond thin films functionalized with therapeutically active collagen networks. J. Phys. Chem. B 2009; 113(10):2966-71. doi:1 0.1021/jp9004086

25. Fitzpatrick JM. The application of nanotechnology for the treatment of metastatic prostate cancer. BJU Int. 2009; 104(6).

26. Xia T, Li N, Nel AE. Potential health impact of nanoparticles. Annu. Rev. Public Health. 2009; 30:137-50. doi:10.1146/annurev.publhealth.031308.100155

27. Xiao Q, Zhou B, Huang S, Tian F, Guan H, Ge Y, Liu X, $\mathrm{He} \mathrm{Z}$, Liu Y. Direct observation of the binding process between protein and quantum dots by in situ surface plasmon resonance measurements. Nanotechnology 2009; 20(32): 325101. doi:10.1088/0957-4484/20/32/325101

28. Li N, Xia T, Nel AE. The role of oxidative stress in ambient particulate matter-induced lung diseases and its implications in the toxicity of engineered nanoparticles. Free Radic Biol. Med. 2008; 44(9):1689-99. doi:10.1016/j.freeradbiom ed.2008.01.028

29. Cai W, Chen X. Multimodality molecular imaging of tumor angiogenesis. J. Nucl. Med. 2008; 49(s2):113s-128s. doi:10.2967/inumed.107.045922

30. Liang XJ, Chen C, Zhao Y, Jia L, Wang PC. Biopharmaceutics and therapeutic potential of engineered nanomaterials. Curr. Drug Metab. 2008; 9(8):697-709. doi:10.2 174/138920008786049230

31. Liu L, Zhang J, Su X, Mason RP.In vitro and In vivo Assessment of CdTe and CdHgTe Toxicity and Clearance. $J$. Biomed. Nanotechnol. 2008; 4(4):524-528. doi:10.1166 /jbn.2008.018

32. Yin JJ, Lao F, Meng J, Fu PP, Zhao Y, Xing G, Gao X, Sun $\mathrm{B}$, Wang PC, Chen C, Liang XJ. Inhibition of tumor growth by endohedral metallofullerenol nanoparticles optimized as reactive oxygen species scavenger. Mol. Pharmacol. 2008; 74(4):1132-40. doi:10.1124/mol.108.048348

33. Tao SL, Desai TA. Microfabricated drug delivery systems: from particles to pores. Adv. Drug Delivery. Rev. 2003; 55:315-28. doi:10.1016/S0169-409X(02)00227-2

34. Zhang MJ, Tarn TJ, Xi N. Micro-/nano-devices for controlled drug delivery. Proceedings of the 2004 IEEE International Conference on Robotics and Automation; 2004 April 26-May 1; New Orleans, La. New Orleans. The Conference; 2004; 2068-73.

35. Krukemeyer MG, Wagne W, Jakobs M, Kren V. Tumor regression by means of magnetic drug targeting. Nanomedicine 2009; 4(8): 875-882. doi:10.2217/nnm.09.73

36. Tong L, Zhao Y, Huff T, Hansen M, Wei A, Cheng J. Gold Nanorods Mediate Tumor Cell Death by Compromising Membrane Integrity. Adv. Mater. 2007; 19: 3136-3141. doi:10.1002/adma.200701974

37. Li Z, Huang P, Zhang X, Lin J, Yang S, Liu B, Gao F, Xi P, Ren QS, Cui DX. RGD-conjugated dendrimer-modified gold nanorods for in Vivo tumor targeting and photothermal therapy. Mol. Pharm. 2010; doi:10.1021/mp9001415

38. Pan B, Ao L, Gao F, Tian H, He R, Cui D. End-to-end selfassembly and colorimetric characterization of gold nanorods and nanospheres via oligonucleotide hybridization. Nanotechnology 2005; 16:1776-1780. doi:10.1088/09574484/16/9/061

39. Pan B; Cui D; Sheng Y; Ozkan C, Gao F, He R, Li Q.; Xu $\mathrm{P}$, Huang T. Dendrimer-modified magnetic nanoparticles enhance efficiency of gene delivery system. Cancer Res. 2007; 67: 8156-8163. doi:10.1158/0008-5472.CAN-064762

40. Cai W, Chen X. Preparation of peptide-conjugated quantum dots for tumor vasculature-targeted imaging. Nat. Protoc. 2008; 3: 89-96. doi:10.1038/nprot.2007.478

41. Song H, He R, Wang K, Ruan J, Bao CC, Li N, Ji JJ, Cui DX. Anti-HIF-1a antibody-conjugated pluronic triblock copolymers encapsulated with Paclitaxel for tumor targeting therapy. Biomaterials 2009, doi:10.1016/j.bio materials.2009.11.067

42. Chen Z, Chen H, Meng H, Xing G, Gao X, Sun B, Shi X, Yuan H, Zhang C, Liu R, Zhao F, Zhao Y, Fang X. Biodistribution and metabolic paths of silica coated CdSeS quantum dots. Toxicol. Appl. Pharmacol. 2008; 230(3):36471. doi:10.1016/j.taap.2008.03.022

43. Tang N, Du G, Wang N, Liu C, Hang H, and Liang W Improving Penetration in Tumors with Nano- assemblies of Phospholipids and Doxorubicin. J. Natl. Cancer Inst. 2007; 99: 1004-1015. doi:10.1093/jnci/djm027

44. Dreher MR, Chilkoti A. Toward a Systems Engineering Approach to Cancer Drug Delivery. J. Natl. Cancer Inst .2007; 99: 983-985. doi:10.1093/jnci/djm042

45. Wei G, Xiao S, Si D, Liu C. Comparative pharmacokinetics of micelle- or liposomal encapsulated doxorubicin formulations to healthy rats and tumor-bearing mice. Drug Metabolism Reviews 2008; 40(S2):81-82.

46. Xu Y, Xiao S, Wei G, Liu C, Si D. Nanoparticle of doxorubicin eliminate the accumulation in tissues of tumorbearing mice. Drug Metabolism Reviews 2008; 40(S2):193.

47. Cheng T, Si D, Liu C. Rapid and sensitive LC-MS method for pharmacokinetic study of vinorelbine in rats. Biomedical Chromatography, 2009; 23:909-911. doi:10.100 2/bmc. 1216

48. Zhang J, Zhang Z, Yang H, Tan Q, Qin S, Qiu X. Lyophilized Paclitaxel Magnetoliposomes as a Potential Drug Delivery System for Breast Carcinoma via Parenteral Administration: In Vitro and in Vivo Studies. Pharmaceutical Research 2005; 22(4):573-583. doi:10.1007/s11095-0052496-8

49. Li Q, Cheng T, Huang Y, Si D, Liu C. Comparative pharmacokinetics and tissue distribution between free and Micelle-Encapsulated Alprostadil. Drug Metabolism Reviews, 2008; 40(S2):80-81.

50. Thomas T, Thomas K, Sadrich N, Savage N, Adair P, Bronaugh R. Research strategies for safety evaluation of nanomaterials, Part VII: Evaluating consumer exposure to nanoscale materials. Toxicol. Sci. 2006; 91(1):14-19. doi:10.1093/toxsci/kfj129

51. Gubbels-van Hal WMLG, Blaauboer BJ, Barentsen HM, 
Hoitink MA, Meerts IATM, van der Hoeven JCM. An alternative approach for the safety evaluation of new and existing chemicals, an exercise in integrated testing. Reg. Tox icol. Pharmacol. 2005; 42:284-295. doi:10.1016/j. yrtph.2005.05.002

52. Cui D, Gao H. Advance and prospect of bionanomaterials Biotechnol. Prog. 2003; 19:683-692. doi:10.1021/bp025 $\underline{791 \mathrm{i}}$
Received 10 November, 2009; accepted 6 December, 2009; published online 9 December, 2009.

Copyright: (c) 2009 C. Liu et al. This is an open-access article distributed under the terms of the Creative Commons Attribution License, which permits unrestricted use, distribution, and reproduction in any medium, provided the original author and source are credited. 